\title{
Effect of Stapling on the Thermodynamics of mdm2-p53 Binding
}

Atanu Maity ${ }^{\dagger}$, Asha Rani Choudhury ${ }^{\dagger}$ and Rajarshi Chakrabarti*

Department of Chemistry, Indian Institute of Technology Bombay, Mumbai, Powai 400076

* rajarshi@chem.iitb.ac.in

(a) $\mathbf{p 5 3}$

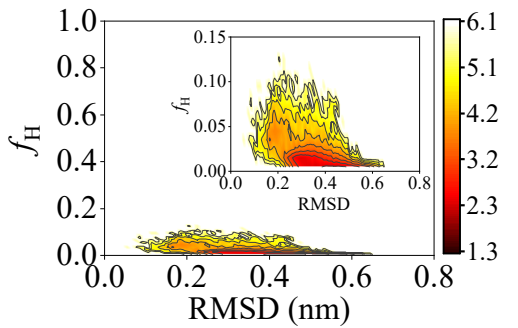

(d) $\mathbf{p 5 3}^{\mathrm{Al}}$

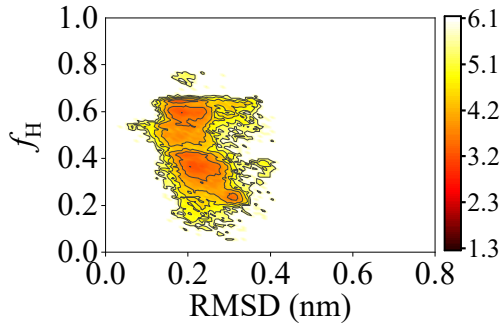

(g) $\mathrm{p53}^{\mathrm{Ar}}$

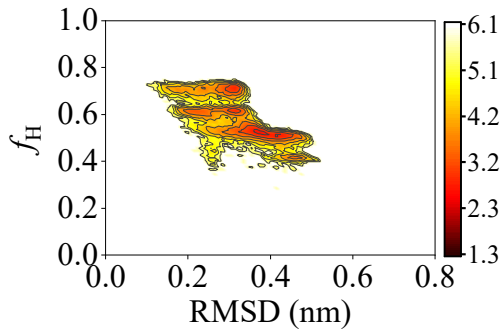

(b) p53_330K

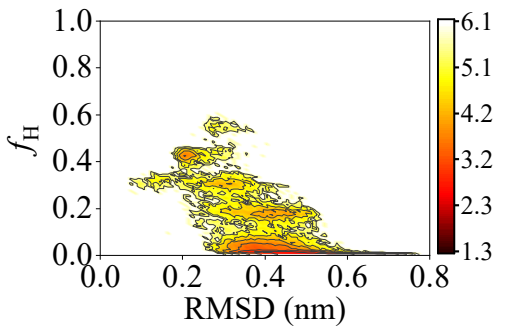

(e) $\mathrm{p53}^{\mathrm{Al}} \_330 \mathrm{~K}$

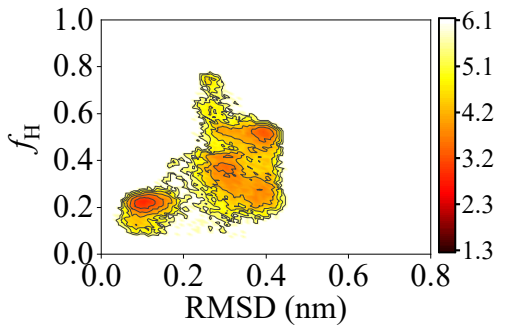

(h) $\mathrm{p53}^{\mathrm{Ar}} \_330 \mathrm{~K}$

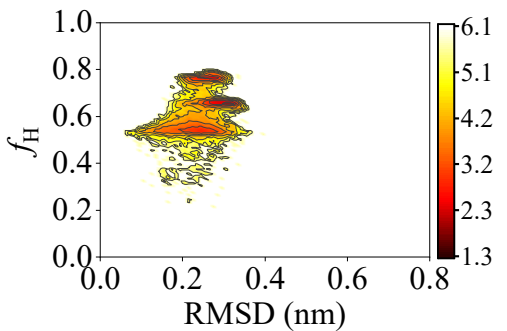

(c) p53 urea

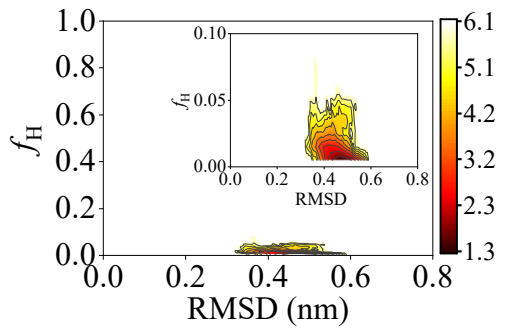

(f) $\mathrm{p53}^{\mathrm{Al}}$ _urea

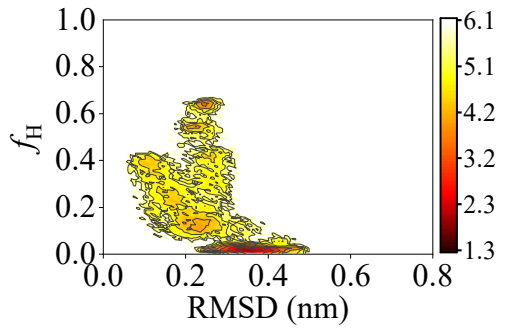

(i) $\mathrm{p}^{\mathrm{Ar}}{ }_{-}$urea

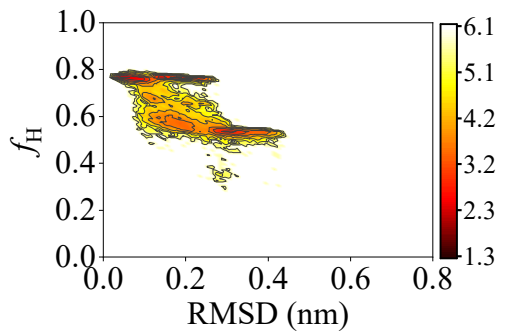

Figure S1: 2D histogram of Root mean square deviation (RMSD) vs helical fraction plot for the p53 peptide and the p53 peptides with aliphatic and aromatic staples in different conditions (i.e. in the aqueous medium, at high temperature, and in the presence of urea) showing variation in conformation in the presence of stapling agents. The dark red-to-yellow-to-white color scale represents increasing conformational free energy in $\mathrm{kcal} / \mathrm{mol}$. 
(a) $\mathbf{p 5 3}$

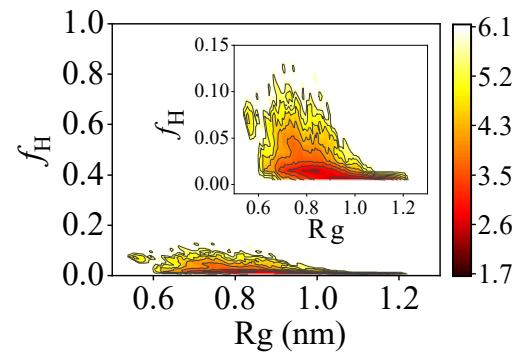

(d) $\mathrm{p53}^{\mathrm{Al}}$

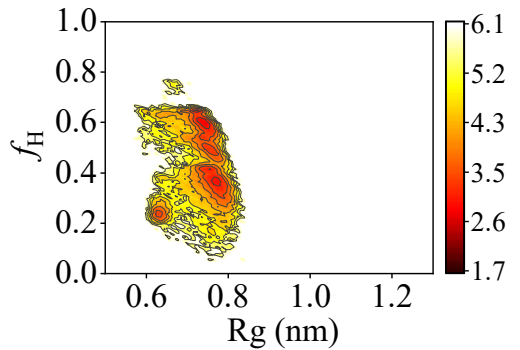

(g) $\mathrm{p53}^{\mathrm{Ar}}$

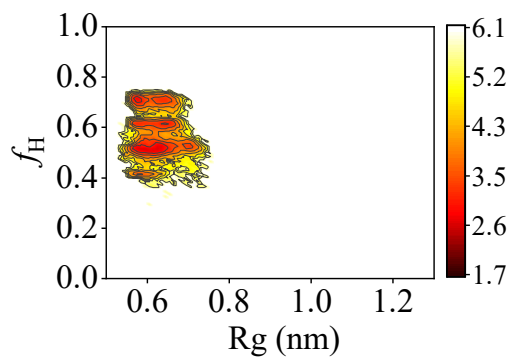

(b) $\mathrm{p53} \_330 \mathrm{~K}$

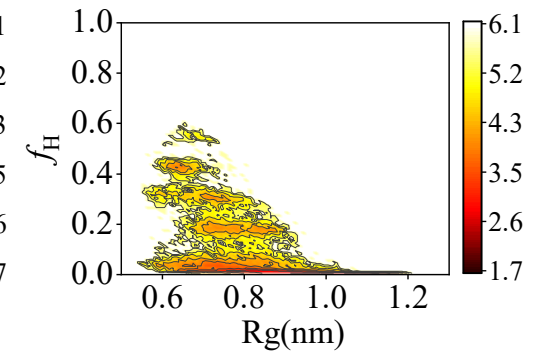

(e) $\mathrm{p}^{\mathrm{Al}}{ }^{\mathrm{Al}}$ _330K

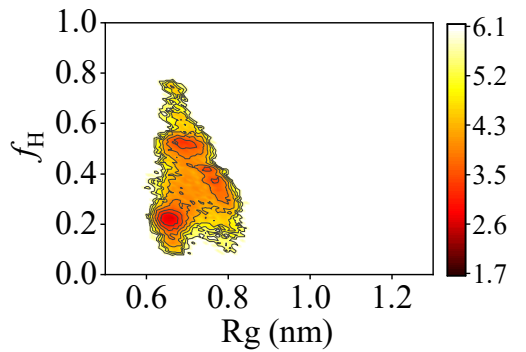

(h) $\mathrm{p53}^{\mathrm{Ar}}{ }_{330 \mathrm{~K}}$

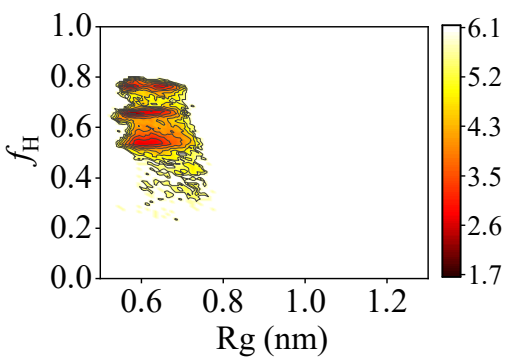

(c) p53_urea

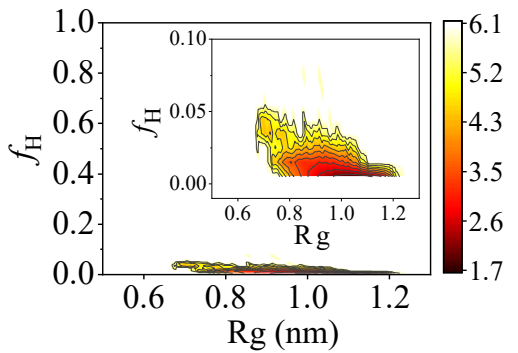

(f) $\mathrm{p}^{\mathrm{Al}}{ }^{\mathrm{Al}}$ urea

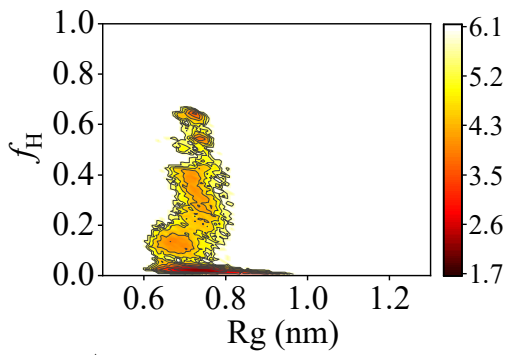

(i) $\mathrm{p53}^{\mathrm{Ar}}$ _urea

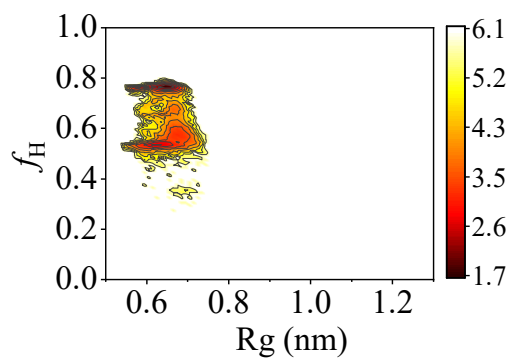

Figure S2: 2D histogram of Radius of gyration $\left(R_{g}\right)$ vs helical fraction plot for the p53 peptide and the p53 peptides with aliphatic and aromatic staples in different conditions (i.e. in the aqueous medium, at high temperature, and in the presence of urea) showing variation in conformation in the presence of stapling agents. The dark red-to-yellow-to-white color scale represents increasing conformational free energy in $\mathrm{kcal} / \mathrm{mol}$.
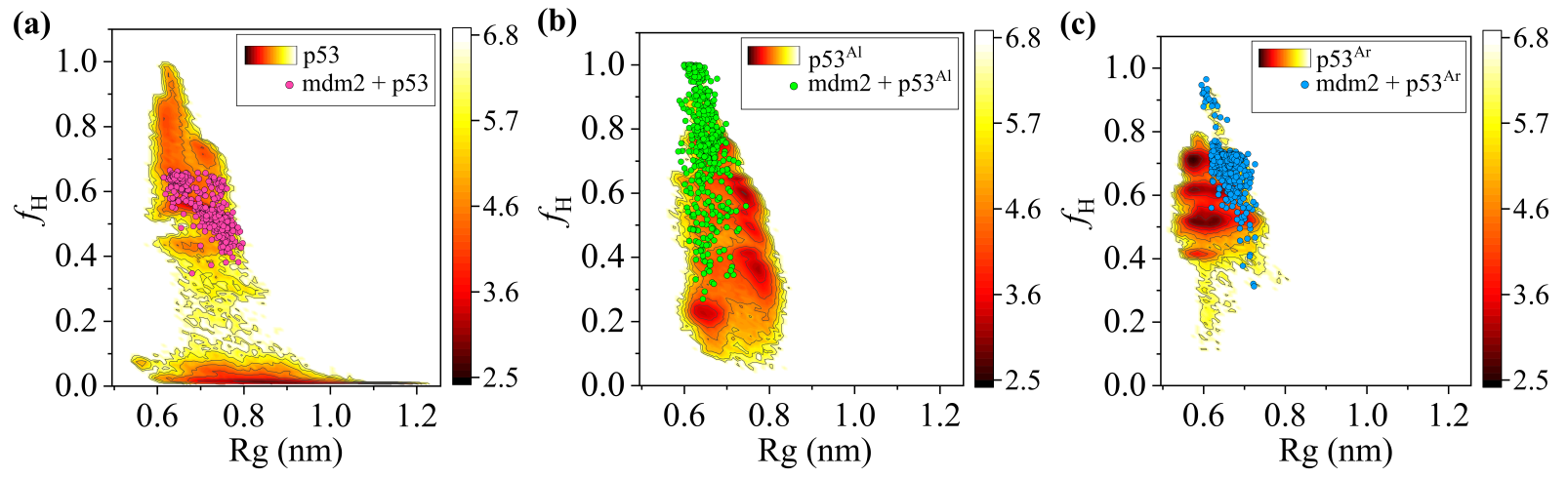

Figure S3: Quantification of unfolded to folded conformational transition during complexation. The $\mathrm{R}_{g}$ and helicity of the conformations of (a) mdm2+p53, (b) mdm2 $+\mathrm{p} 53^{\mathrm{Al}}$, and (c) $\mathrm{mdm} 2+\mathrm{p} 53^{\mathrm{Ar}}$ system are plotted on the $R_{g}$ vs helicity free energy surfaces of $\mathrm{p} 53, \mathrm{p} 53^{\mathrm{Al}}$ and $\mathrm{p} 53^{\mathrm{Ar}}$ respectively. There is a large energy gap between the energy minimum of $\mathrm{p} 53$ and the points corresponding to $\mathrm{mdm} 2+\mathrm{p} 53$ (magenta circles) whereas there is a considerable overlap between the energy minima for $\mathrm{p} 53^{\mathrm{Al}}$ and $\mathrm{p} 53^{\mathrm{Ar}}$ with the points corresponding to $\mathrm{p} 53^{\mathrm{Al}}$ (green circles) and $\mathrm{p} 53^{\mathrm{Ar}}$ (cyan circles) respectively. The dark red-to-yellow-to-white color scale represents increasing conformational free energy in $\mathrm{kcal} / \mathrm{mol}$. 

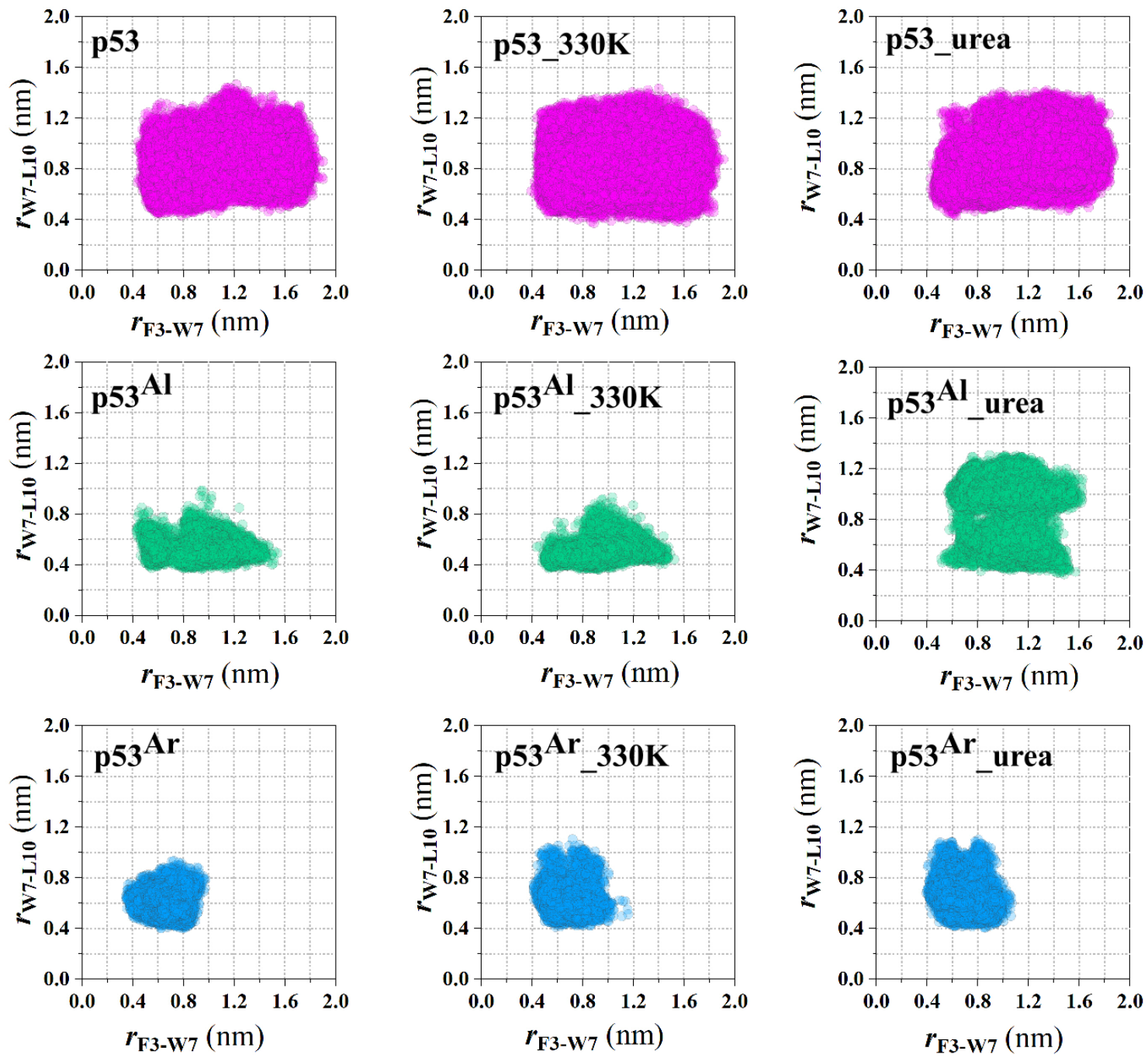

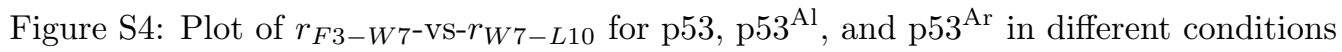

Table S1: Energy components for the three simulations of $\mathbf{m d m 2}$. All energy values and standard errors are in $\mathrm{kcal} / \mathrm{mol}$.

\begin{tabular}{|l|r|r|r|r|}
\hline $\begin{array}{c}\text { Energy } \\
\text { components }\end{array}$ & \multicolumn{1}{c|}{$\begin{array}{c}\text { mdm2 } \\
\text { (simulation 1) }\end{array}$} & $\begin{array}{c}\text { mdm2 } \\
\text { (simulation 2) }\end{array}$ & $\begin{array}{c}\text { mdm2 } \\
\text { (simulation 3) }\end{array}$ & \multicolumn{1}{c|}{ Average } \\
\hline$E_{\text {Elec }}$ & $-1550.90 \pm 203.51$ & $-1607.59 \pm 198.50$ & $-1574.90 \pm 198.22$ & $-1577.80 \pm 23.23$ \\
\hline$E_{V d w}$ & $-296.42 \pm 15.91$ & $-285.81 \pm 15.43$ & $-287.13 \pm 15.19$ & $-289.79 \pm 4.72$ \\
\hline$E_{\text {Internal }}$ & $1986.60 \pm 29.15$ & $1981.54 \pm 29.12$ & $1982.92 \pm 28.94$ & $1983.69 \pm 2.14$ \\
\hline$E_{\text {Solv (polar) }}$ & $-1558.44 \pm 198.94$ & $-1501.07 \pm 194.03$ & $-1535.24 \pm 193.27$ & $-1531.58 \pm 23.56$ \\
\hline$E_{\text {Solv(non-polar) }}$ & $33.06 \pm 0.67$ & $33.37 \pm 0.63$ & $33.58 \pm 0.59$ & $33.34 \pm 0.21$ \\
\hline$E_{\text {Elec } \text { Solv(polar })}$ & $-3109.34 \pm 16.47$ & $-3108.66 \pm 15.94$ & $-3110.98 \pm 16.25$ & $-3109.38 \pm 0.6$ \\
\hline$H$ & $-1386.10 \pm 28.74$ & $-1379.56 \pm 28.45$ & $-1380.77 \pm 27.85$ & $-1382.14 \pm 2.84$ \\
\hline$-T S$ & $-355.40 \pm 0.53$ & $-369.71 \pm 0.17$ & $-378.14 \pm 0.36$ & $-367.75 \pm 9.39$ \\
\hline
\end{tabular}


Table S2: Energy components for the three simulations of $\mathbf{m d m} \mathbf{2}+\mathbf{p} 53$. All energy values and standard errors are in $\mathrm{kcal} / \mathrm{mol}$.

\begin{tabular}{|l|r|r|r|r|}
\hline $\begin{array}{c}\text { Energy } \\
\text { components }\end{array}$ & \multicolumn{1}{c|}{$\begin{array}{c}\text { mdm2+p53 } \\
\text { (simulation 1) }\end{array}$} & $\begin{array}{c}\text { mdm2+p53 } \\
\text { (simulation 2) }\end{array}$ & $\begin{array}{c}\text { mdm2+p53 } \\
\text { (simulation 3) }\end{array}$ & \multicolumn{1}{c|}{ Average } \\
\hline$E_{\text {Elec }}$ & $-1868.45 \pm 223.07$ & $-1765.45 \pm 227.80$ & $-1971.43 \pm 221.30$ & $-1868.44 \pm 84.09$ \\
\hline$E_{V d w}$ & $-364.14 \pm 16.75$ & $-358.39 \pm 17.02$ & $-357.21 \pm 17.42$ & $-359.91 \pm 3.03$ \\
\hline$E_{\text {Internal }}$ & $2303.48 \pm 31.10$ & $2305.99 \pm 31.36$ & $2310.67 \pm 30.93$ & $2306.71 \pm 2.98$ \\
\hline$E_{\text {Solv (polar })}$ & $-1732.36 \pm 218.20$ & $-1842.15 \pm 222.23$ & $-1644.16 \pm 216.22$ & $-1739.56 \pm 80.99$ \\
\hline$E_{\text {Solv(non-polar })}$ & $37.17 \pm 0.82$ & $37.76 \pm 0.83$ & $37.48 \pm 0.81$ & $37.47 \pm 0.24$ \\
\hline$E_{\text {Elec }+ \text { Solv(polar })}$ & $-3600.81 \pm 17.77$ & $-3607.60 \pm 18.0$ & $-3615.59 \pm 17.48$ & $-3608.00 \pm 6.04$ \\
\hline$H$ & $-1624.29 \pm 30.66$ & $-1622.24 \pm 31.37$ & $-1624.65 \pm 30.38$ & $-1623.73 \pm 1.06$ \\
\hline$-T S$ & $-429.40 \pm 1.13$ & $-431.40 \pm 2.69$ & $-427.55 \pm 0.91$ & $-429.45 \pm 1.57$ \\
\hline
\end{tabular}

Table S3: Energy components for the three simulations of $\mathbf{m d m 2}+\mathbf{p 5 3} \mathbf{3}^{\mathbf{A l}}$. All energy values and standard errors are in $\mathrm{kcal} / \mathrm{mol}$.

\begin{tabular}{|l|r|r|r|r|}
\hline $\begin{array}{c}\text { Energy } \\
\text { components }\end{array}$ & \multicolumn{1}{c|}{$\begin{array}{c}\text { mdm2 }+\mathrm{p} 53^{\mathrm{AI}} \\
(\text { simulation 1) }\end{array}$} & $\begin{array}{c}\text { mdm2 }+\mathrm{p} 53^{\mathrm{Al}} \\
(\text { simulation 2) }\end{array}$ & $\begin{array}{c}\text { mdm2 }+\mathrm{p} 53^{\mathrm{Al}} \\
(\text { simulation 3) }\end{array}$ & \multicolumn{1}{c|}{ Average } \\
\hline$E_{\text {Elec }}$ & $-1866.26 \pm 227.91$ & $-1943.48 \pm 229.18$ & $-1920.53 \pm 218.78$ & $-1910.09 \pm 32.38$ \\
\hline$E_{\text {Vdw }}$ & $-374.87 \pm 17.53$ & $-374.46 \pm 17.02$ & $-372.92 \pm 17.23$ & $-374.08 \pm 0.84$ \\
\hline$E_{\text {Internal }}$ & $2345.72 \pm 31.39$ & $2341.94 \pm 31.67$ & $2341.03 \pm 31.51$ & $2342.90 \pm 2.03$ \\
\hline$E_{\text {Solv (polar })}$ & $-1765.86 \pm 223.54$ & $-1683.43 \pm 223.50$ & $-1709.17 \pm 213.80$ & $-1719.49 \pm 34.43$ \\
\hline$E_{\text {Solv (non-polar })}$ & $37.18 \pm 0.76$ & $37.11 \pm 0.89$ & $37.20 \pm 0.81$ & $37.17 \pm 0.04$ \\
\hline$E_{\text {Elec }+ \text { Solv (polar })}$ & $-3632.12 \pm 17.57$ & $-3626.91 \pm 18.41$ & $-3629.70 \pm 17.70$ & $-3629.58 \pm 2.13$ \\
\hline$H$ & $-1624.09 \pm 31.73$ & $-1622.31 \pm 31.85$ & $-1624.39 \pm 30.96$ & $-1623.60 \pm 0.92$ \\
\hline$-T S$ & $-423.68 \pm 0.18$ & $-432.45 \pm 0.48$ & $-425.12 \pm 0.17$ & $-427.08 \pm 3.84$ \\
\hline
\end{tabular}

Table S4: Energy components for the three simulations of $\mathbf{m d m} \mathbf{2}+\mathbf{p} 5 \mathbf{3}^{\mathbf{A r}}$. All energy values and standard errors are in $\mathrm{kcal} / \mathrm{mol}$.

\begin{tabular}{|l|r|r|r|r|}
\hline $\begin{array}{c}\text { Energy } \\
\text { components }\end{array}$ & \multicolumn{1}{c|}{$\begin{array}{c}\text { mdm2 }+\mathrm{p} 53^{\mathrm{Ar}} \\
(\text { simulation } 1)\end{array}$} & $\begin{array}{c}\text { mdm2 }+\mathrm{p} 53^{\mathrm{Ar}} \\
(\text { simulation 2) }\end{array}$ & $\begin{array}{c}\text { mdm2 }+\mathrm{p} 53^{\mathrm{Ar}} \\
(\text { simulation 3) }\end{array}$ & \multicolumn{1}{c|}{ Average } \\
\hline$E_{\text {Elec }}$ & $-1903.94 \pm 224.35$ & $-1972.60 \pm 218.79$ & $-1955.18 \pm 222.07$ & $-1943.91 \pm 29.14$ \\
\hline$E_{V d w}$ & $-367.50 \pm 17.75$ & $-362.22 \pm 16.22$ & $-364.20 \pm 17.43$ & $-364.64 \pm 2.18$ \\
\hline$E_{\text {Internal }}$ & $2324.47 \pm 31.66$ & $2324.41 \pm 31.23$ & $2327.33 \pm 31.35$ & $2325.40 \pm 1.36$ \\
\hline$E_{\text {Solv }(\text { polar })}$ & $-1725.82 \pm 219.08$ & $-1661.12 \pm 214.53$ & $-1677.61 \pm 217.47$ & $-1688.18 \pm 27.45$ \\
\hline$E_{\text {Solv (non-polar })}$ & $36.97 \pm 0.88$ & $37.16 \pm 0.73$ & $37.01 \pm 0.82$ & $37.05 \pm 0.08$ \\
\hline$E_{\text {Elec }+ \text { Solv (polar })}$ & $-3629.76 \pm 18.29$ & $-3633.72 \pm 18.39$ & $-3632.79 \pm 17.81$ & $-3632.09 \pm 1.69$ \\
\hline$H$ & $-1635.83 \pm 31.28$ & $-1634.36 \pm 31.59$ & $-1632.65 \pm 31.00$ & $-1634.28 \pm 1.30$ \\
\hline$-T S$ & $-429.89 \pm 1.12$ & $-431.72 \pm 1.40$ & $-417.48 \pm 0.48$ & $-426.36 \pm 6.32$ \\
\hline
\end{tabular}

Table S5: Energy components for the three simulations of p53. All energy values and standard errors are in kcal $/ \mathrm{mol}$.

\begin{tabular}{|l|r|r|r|r|}
\hline $\begin{array}{c}\text { Energy } \\
\text { components }\end{array}$ & \multicolumn{1}{c|}{$\begin{array}{c}\text { p53 } \\
\text { (simulation 1) }\end{array}$} & $\begin{array}{c}\text { p53 } \\
\text { (simulation 2) }\end{array}$ & $\begin{array}{c}\text { p53 } \\
\text { (simulation 3) }\end{array}$ & \multicolumn{1}{c|}{ Average } \\
\hline$E_{\text {Elec }}$ & $-110.78 \pm 51.21$ & $-113.94 \pm 47.87$ & $-118.82 \pm 53.20$ & $-114.51 \pm 3.31$ \\
\hline$E_{V d w}$ & $3.06 \pm 6.16$ & $1.17 \pm 8.60$ & $-1.34 \pm 8.52$ & $0.96 \pm 1.80$ \\
\hline$E_{\text {Internal }}$ & $309.50 \pm 13.48$ & $310.67 \pm 11.88$ & $311.52 \pm 11.74$ & $310.57 \pm 0.83$ \\
\hline$E_{\text {Solv(polar) }}$ & $-387.99 \pm 48.66$ & $-385.21 \pm 45.41$ & $-381.25 \pm 49.39$ & $-384.81 \pm 2.76$ \\
\hline$E_{\text {Solv (non-polar) }}$ & $8.69 \pm 0.35$ & $8.54 \pm 0.53$ & $8.42 \pm 0.56$ & $8.55 \pm 0.11$ \\
\hline$E_{\text {Elec }+ \text { Solv(polar) }}$ & $-498.76 \pm 6.56$ & $-499.15 \pm 6.53$ & $-500.07 \pm 7.76$ & $-499.32 \pm 0.55$ \\
\hline$H$ & $-177.51 \pm 14.20$ & $-178.76 \pm 13.34$ & $-181.47 \pm 14.59$ & $-179.25 \pm 1.65$ \\
\hline$-T S$ & $-114.02 \pm 0.07$ & $-116.63 \pm 0.54$ & $-116.09 \pm 0.11$ & $-115.58 \pm 1.12$ \\
\hline
\end{tabular}


Table S6: Energy components for the three simulations of $\mathbf{p 5 3} \mathbf{A l}^{\mathbf{A l}}$. All energy values and standard errors are in $\mathrm{kcal} / \mathrm{mol}$.

\begin{tabular}{|c|c|c|c|c|}
\hline $\begin{array}{c}\text { Energy } \\
\text { components }\end{array}$ & $\begin{array}{c}\mathrm{p} 53^{\mathrm{Al}} \\
\text { (simulation 1) }\end{array}$ & $\begin{array}{c}\mathrm{p} 53^{\mathrm{Al}} \\
\text { (simulation 2) }\end{array}$ & $\begin{array}{c}\mathrm{p} 53^{\mathrm{Al}} \\
\text { (simulation 3) }\end{array}$ & $\mathrm{Av}$ \\
\hline$E_{\text {Elec }}$ & $-188.58 \pm 49.43$ & $-165.13 \pm 40.75$ & $-227.12 \pm 41.20$ & $193.61=$ \\
\hline$E_{V}$ & $-15.63 \pm 5$ & $3 \pm 5$ & $-15.74 \exists$ & -1 \\
\hline & 14.4 & $345.87 \pm$ & $348.08 \pm 12.23$ & 346 \\
\hline & $35.37 \pm 45.76$ & $-354.60 \pm 39.30$ & $-301.30 \pm 38.43$ & $-330.42 \pm 22.04$ \\
\hline & 8.10 & $8.32 \pm 0.24$ & $7.93 \pm 0.22$ & 0.16 \\
\hline & $-523.95=$ & $-519.73 \pm 6.97$ & $-528.41 \pm 6.69$ & $-524.03 \pm 3.54$ \\
\hline$H$ & $187.03 \pm 15.35$ & $179.62 \pm 12.34$ & $-188.14 \pm 11.91$ & $-184.93 \pm 3.78$ \\
\hline$-T S$ & $-86.38 \pm 0.43$ & $-91.53 \pm 0.84$ & $-79.31 \pm 0.17$ & $-85.74 \pm 5.01$ \\
\hline
\end{tabular}

Table S7: Energy components for the three simulations of $\mathbf{p 5 3}{ }^{\mathbf{A r}}$. All energy values and standard errors are in $\mathrm{kcal} / \mathrm{mol}$.

\begin{tabular}{|c|c|c|c|c|}
\hline $\begin{array}{c}\text { Energy } \\
\text { components }\end{array}$ & $\begin{array}{c}\mathrm{p} 53^{\mathrm{Ar}} \\
\text { (simulation 1) }\end{array}$ & $\begin{array}{c}\mathrm{p} 53^{\mathrm{Ar}} \\
\text { (simulation 2) }\end{array}$ & $\begin{array}{c}\mathrm{p} 53^{\mathrm{Ar}} \\
\text { (simulation 3) }\end{array}$ & Average \\
\hline$E_{\text {Elec }}$ & $-183.83 \pm 38.21$ & $-183.10 \pm 38.54$ & $-188.74 \pm 38.37$ & $-185.22 \pm 2.50$ \\
\hline$E_{V d w}$ & $-7.04 \pm 7.14$ & $-7.65 \pm 7.22$ & $-8.65 \pm 6.80$ & $-7.78 \pm 0.66$ \\
\hline$E_{\text {Inter }}$ & $330.74 \pm 12.01$ & $333.37 \pm 11.93$ & $333.52 \pm 11.88$ & $332.68 \pm 1.38$ \\
\hline$E_{\text {Solv }(p o}$ & $-345.11 \pm 36.04$ & $-346.99 \pm 36.59$ & $-342.13 \pm 36.80$ & $-344.80 \pm 1.92$ \\
\hline$E_{\text {Solv }(n c}$ & $7.67 \pm 0.32$ & $7.69 \pm 0.30$ & $7.56 \pm 0.30$ & $7.64 \pm 0.06$ \\
\hline$E_{\text {Elec }+ \text { Solv }}$ & $-528.94 \pm 6.66$ & $-530.09 \pm 6.37$ & $-531.05 \pm 6.05$ & $-530.03 \pm 0.86$ \\
\hline$H$ & $-197.58 \pm 11.91$ & $-196.27 \pm 12.36$ & $-198.61 \pm 12.01$ & $-197.49 \pm 0.96$ \\
\hline$-T S$ & $-77.29 \pm 0.63$ & $-72.60 \pm 0.28$ & $-73.82 \pm 0.21$ & $-74.57 \pm 1.99$ \\
\hline
\end{tabular}

Table S8: Binding free energy of mdm2-p53 binding from simulation 1 . All energy values are in $\mathrm{kcal} / \mathrm{mol}$.

\begin{tabular}{|c|c|c|c|c|}
\hline $\begin{array}{c}\text { Energy } \\
\text { components }\end{array}$ & $\mathrm{mdm} 2+\mathrm{p} 53$ & $\mathrm{mdm} 2$ & $\mathrm{p} 53$ & $\Delta E$ \\
\hline$E_{\text {Elec }}$ & $-1868.44 \pm 84.09$ & $-1577.80 \pm 23.23$ & $-114.51 \pm 3.31$ & -176.14 \\
\hline$E_{V d w}$ & $-359.91 \pm 3.03$ & $-289.79 \pm 4.72$ & $0.96 \pm 1.80$ & -71.09 \\
\hline$E_{\text {Internal }}$ & $2306.71 \pm 2.98$ & $1983.69 \pm 2.14$ & $310.57 \pm 0.83$ & 12.46 \\
\hline$E_{\text {Solv(polar })}$ & $-1739.56 \pm 80.99$ & $-1531.58 \pm 23.56$ & $-384.81 \pm 2.76$ & 176.84 \\
\hline$E_{\text {Solv }(\text { non-polar })}$ & $37.47 \pm 0.24$ & $33.34 \pm 0.21$ & $8.55 \pm 0.11$ & -4.41 \\
\hline$E_{\text {Elec }+ \text { Solv }(\text { polar })}$ & $-3608.00 \pm 6.04$ & $-3109.38 \pm 0.60$ & $-499.32 \pm 0.55$ & 0.70 \\
\hline$H$ & $-1623.73 \pm 1.06$ & $-1382.14 \pm 2.84$ & $-179.26 \pm 1.65$ & -62.34 \\
\hline$-T S$ & $-429.45 \pm 1.57$ & $-367.75 \pm 9.39$ & $-115.58 \pm 1.39$ & 53.88 \\
\hline$G$ & & & & $-8.46 \pm 0.69$ \\
\hline
\end{tabular}

Table S9: Binding free energy of $\mathbf{m d m} \mathbf{2}+\mathbf{p} 5 \mathbf{3}^{\mathrm{Al}}$ binding from simulation 1 . All energy values are in $\mathrm{kcal} / \mathrm{mol}$.

\begin{tabular}{|c|c|c|c|c|}
\hline $\begin{array}{c}\text { Energy } \\
\text { components }\end{array}$ & $\mathrm{mdm} 2+\mathrm{p} 53^{\mathrm{Al}}$ & mdm2 & $\mathrm{p} 53^{\mathrm{Al}}$ & $\Delta E$ \\
\hline$E_{\text {Elec }}$ & $-1910.91 \pm 32.38$ & $1577.80 \pm 23.23$ & $-193.61 \pm 25.56$ & -138.69 \\
\hline$E_{V d w}$ & $-374.08 \pm 0.84$ & $-289.79 \pm 4.72$ & $-15.15 \pm 0.76$ & -69.14 \\
\hline$E_{\text {Inter }}$ & $2342.90 \pm 2.03$ & $1983.69 \pm 2.14$ & $346.14 \pm 1.49$ & 13.07 \\
\hline$E_{\text {Solv (pola }}$ & $-1719.49 \pm 34.43$ & $-1531.58 \pm 23.56$ & $-330.42 \pm 22.04$ & 142.52 \\
\hline$E_{\text {Solv }(r}$ & $37.17 \pm 0.04$ & $33.34 \pm 0.21$ & $8.12 \pm 0.16$ & -4.29 \\
\hline$E_{\text {Elec }+ \text { Solv }(\text { polar }}$ & $-3629.58 \pm 2.13$ & $-3109.38 \pm 0.60$ & $-524.03 \pm 3.54$ & 3.83 \\
\hline$H$ & $-1623.60 \pm 0.92$ & $-1382.14 \pm 2.84$ & $-184.93 \pm 3.78$ & -56.53 \\
\hline$-T S$ & $-427.08 \pm 3.84$ & $-367.75 \pm 9.39$ & $-85.74 \pm 5.01$ & 26.41 \\
\hline$G$ & & & & $-30.12 \pm 2.97$ \\
\hline
\end{tabular}


Table S10: Binding free energy of $\mathbf{m d m 2}+\mathbf{p 5 3} \mathbf{3}^{\mathbf{A r}}$ binding from simulation 1 . All energy values are in $\mathrm{kcal} / \mathrm{mol}$.

\begin{tabular}{|l|r|r|r|r|}
\hline $\begin{array}{c}\text { Energy } \\
\text { components }\end{array}$ & \multicolumn{1}{c|}{$\mathrm{mdm2}+\mathrm{p} 53^{\mathrm{Ar}}$} & \multicolumn{1}{c|}{$\mathrm{mdm} 2$} & \multicolumn{1}{c|}{$\mathrm{p} 53^{\mathrm{Ar}}$} & \multicolumn{1}{c|}{$\Delta E$} \\
\hline$E_{\text {Elec }}$ & $-1943.91 \pm 29.14$ & $-1577.80 \pm 23.23$ & $-185.22 \pm 2.50$ & -180.89 \\
\hline$E_{\text {Vdw }}$ & $-364.64 \pm 2.18$ & $-289.79 \pm 4.72$ & $-7.78 \pm 0.66$ & -67.07 \\
\hline$E_{\text {Internal }}$ & $2325.40 \pm 1.36$ & $1983.69 \pm 2.14$ & $332.68 \pm 1.38$ & 9.04 \\
\hline$E_{\text {Solv (polar) }}$ & $-1688.18 \pm 27.45$ & $-1531.58 \pm 23.56$ & $-344.80 \pm 1.92$ & 188.20 \\
\hline$E_{\text {Solv(non-polar })}$ & $37.05 \pm 0.08$ & $33.34 \pm 0.21$ & $7.64 \pm 0.06$ & -3.93 \\
\hline$E_{\text {Elec }+ \text { Solv(polar })}$ & $-3632.09 \pm 1.69$ & $-3109.38 \pm 0.60$ & $-530.03 \pm 0.86$ & 7.32 \\
\hline$H$ & $-1634.28 \pm 1.30$ & $-1382.14 \pm 2.84$ & $-197.49 \pm 0.96$ & -54.65 \\
\hline$-T S$ & $-426.36 \pm 6.32$ & $-367.75 \pm 9.39$ & $-74.57 \pm 1.99$ & 15.95 \\
\hline$G$ & & & & $-38.70 \pm 2.46$ \\
\hline
\end{tabular}

